ANNALES

POLONICI MATHEMATICI

$81.2(2003)$

\title{
On oscillation of solutions of forced nonlinear neutral differential equations of higher order II
}

\author{
by N. PARHi and R. N. RAth (Berhampur)
}

Abstract. Sufficient conditions are obtained so that every solution of

$$
[y(t)-p(t) y(t-\tau)]^{(n)}+Q(t) G(y(t-\sigma))=f(t)
$$

where $n \geq 2, p, f \in C([0, \infty), \mathbb{R}), Q \in C([0, \infty),[0, \infty)), G \in C(\mathbb{R}, \mathbb{R}), \tau>0$ and $\sigma \geq 0$, oscillates or tends to zero as $t \rightarrow \infty$. Various ranges of $p(t)$ are considered. In order to accommodate sublinear cases, it is assumed that $\int_{0}^{\infty} Q(t) d t=\infty$. Through examples it is shown that if the condition on $Q$ is weakened, then there are sublinear equations whose solutions tend to $\pm \infty$ as $t \rightarrow \infty$.

1. Introduction. In this paper, we study oscillatory and asymptotic behaviour of solutions of

$$
[y(t)-p(t) y(t-\tau)]^{(n)}+Q(t) G(y(t-\sigma))=f(t),
$$

where $n \geq 2, p, f \in C([0, \infty), \mathbb{R}), Q \in C([0, \infty),[0, \infty)), G \in C(\mathbb{R}, \mathbb{R}), \tau>0$ and $\sigma \geq 0$. In [5], most of the results hold for $G$ satisfying

$$
\liminf _{|u| \rightarrow \infty} G(u) / u>\lambda>0 .
$$

We remove this restriction on $G$ in the present work. In most of our results here we assume

$$
\int_{0}^{\infty} Q(t) d t=\infty .
$$

The technique employed in this paper is motivated by Lemma 2.1 (see Section 2). The results hold true for homogeneous equations associated with (1).

By a solution of (1) we mean a real-valued continuous function $y$ on $\left[T_{y}-\varrho, \infty\right)$ for some $T_{y} \geq 0$, where $\varrho=\max \{\tau, \sigma\}$, such that $y(t)-p(t) y(t-\tau)$

2000 Mathematics Subject Classification: 34C10, 34C15, 34K40.

Key words and phrases: oscillation, nonoscillation, neutral equations, asymptotic behaviour. 
is $n$-times continuously differentiable and (1) is satisfied for $t \in\left[T_{y}, \infty\right)$. A solution of (1) is said to be oscillatory if it has arbitrarily large zeros; otherwise, it is called nonoscillatory.

2. Main results. The following assumptions are needed below:

$\left(\mathrm{H}_{2}\right) \quad G$ is nondecreasing and $u G(u)>0$ for $u \neq 0$.

$\left(\mathrm{H}_{3}\right) \quad$ There exists $F \in C^{n}([0, \infty), \mathbb{R})$ such that $\lim _{t \rightarrow \infty} F(t)=0$ and $F^{(n)}(t)=f(t)$.

$\left(\mathrm{H}_{4}\right) \quad$ For $u>0$ and $v>0$, there exists $\alpha>0$ such that $G(u)+G(v) \geq$ $\alpha G(u+v)$.

$\left(\widetilde{\mathrm{H}}_{4}\right) \quad$ For $x<0$ and $y<0$ there exists $\beta>0$ such that $G(x)+G(y) \leq$ $\beta G(x+y)$.

$\left(\mathrm{H}_{5}\right) \quad G(u v)=G(u) G(v)$.

$\left(\mathrm{H}_{6}\right) \quad \int_{\tau}^{\infty} Q^{*}(t) d t=\infty$, where $Q^{*}(t)=\min \{Q(t), Q(t-\tau)\}$.

Further, $p(t)$ satisfies one of the following conditions:

$\left(\mathrm{A}_{1}\right) \quad 0 \leq p(t) \leq p_{1}<1$

$\left(\mathrm{A}_{2}\right) \quad-1<-p_{2} \leq p(t) \leq 0$,

$\left(\mathrm{A}_{3}\right) \quad-p_{3} \leq p(t) \leq 0$,

$\left(\mathrm{A}_{4}\right) \quad p(t)$ changes sign with $-1<-p_{4} \leq p(t) \leq p_{5}<1$ such that $p_{4}+p_{5}$ $<1$, where $p_{4}>0$ and $p_{5}>0$,

$\left(\mathrm{A}_{5}\right) \quad 1 \leq p(t) \leq p_{6}$,

$\left(\mathrm{A}_{6}\right) \quad 0 \leq p(t) \leq p_{7}$.

REMARK 1. The prototype of a function $G$ satisfying $\left(\mathrm{H}_{1}\right)-\left(\widetilde{\mathrm{H}}_{4}\right)$ is $G(u)$ $=|u|^{\gamma} \operatorname{sgn} u, \gamma>0$ (see $\left[4\right.$, p. 292]). Note that $\left(\mathrm{H}_{2}\right)$ and $\left(\mathrm{H}_{5}\right)$ imply that $G(-u)=-G(u)$. Further, $\left(\mathrm{H}_{4}\right)$ and $G(-u)=-G(u)$ imply $\left(\widetilde{\mathrm{H}}_{4}\right)$.

We need the following lemma.

Lemma 2.1 ([3, p. 19]). Let $F, G, p \in C\left(\left[t_{0}, \infty\right), \mathbb{R}\right), t_{0} \geq 0$, be such that $F(t)=G(t)-p(t) G(t-\tau), t \geq t_{0}+\varrho, \tau \geq 0, G(t) \neq 0$ for $t \geq t_{0}$, $\lim \inf _{t \rightarrow \infty} G(t)=0$ if $G(t)>0$ or $\limsup _{t \rightarrow \infty} G(t)=0$ if $G(t)<0$, and $\lim _{t \rightarrow \infty} F(t)=L$ exists. If $p(t)$ satisfies $\left(\mathrm{A}_{i}\right), i=1,2,5,6$, then $L=0$.

THEOREM 2.2. Let $\left(\mathrm{H}_{1}\right),\left(\mathrm{H}_{2}\right)$ and $\left(\mathrm{H}_{3}\right)$ hold. If $p(t)$ satisfies either $\left(\mathrm{A}_{1}\right)$ or $\left(\mathrm{A}_{2}\right)$, then every solution of (1) oscillates or tends to zero as $t \rightarrow \infty$.

Proof. Let $y(t)$ be a nonoscillatory solution of (1). Then $y(t)>0$ or $y(t)<0$ for $t \geq T_{0}>T_{y} \geq 0$. Let $y(t)>0$ for $t \geq T_{0}$. For $t \geq T_{1}>T_{0}+\varrho$, we set

$$
z(t)=y(t)-p(t) y(t-\tau), \quad w(t)=z(t)-F(t) .
$$

From (1) it follows that

$$
w^{(n)}(t)=-Q(t) G(y(t-\sigma)) \leq 0
$$


for $t \geq T_{1}$. Hence, for large $t$, each of $w, w^{\prime}, w^{\prime \prime}, \ldots, w^{(n-1)}$ is monotonic and of constant sign.

We claim that $y(t)$ is bounded. If not, then there exists a strictly increasing sequence $\left\{t_{n}\right\} \subset\left[T_{4}, \infty\right)$ satisfying

$$
\lim _{n \rightarrow \infty} t_{n}=\infty, \quad \lim _{n \rightarrow \infty} y\left(t_{n}\right)=\infty, \quad y\left(t_{n}\right)=\max \left\{y(t): t \in\left[T_{1}, t_{n}\right]\right\} .
$$

From (2) we obtain

$$
w\left(t_{n}\right)=y\left(t_{n}\right)-p\left(t_{n}\right) y\left(t_{n}-\tau\right)-F\left(t_{n}\right) .
$$

Assume $\left(\mathrm{A}_{1}\right)$ holds. Then from $(5)$ we get $w\left(t_{n}\right)>\left(1-p_{1}\right) y\left(t_{n}\right)-F\left(t_{n}\right)$ for $n$ large enough such that $t_{n}-\tau>T_{1}$. Thus $\lim _{n \rightarrow \infty} w\left(t_{n}\right)=\infty$. Consequently, $w(t)>0$ and $w^{\prime}(t)>0, t \geq T_{2}>T_{1}$. From (3) it follows that $w^{(n-1)}(t)>0, t \geq T_{3}>T_{2}$. On the other hand, for $0<\varepsilon<w\left(T_{3}\right),|F(t)|<\varepsilon$ for $t \geq T_{4}>T_{3}$. Hence, for $t \geq T_{4}, w(t)<y(t)-F(t)<y(t)+\varepsilon$, which implies that $0<w\left(T_{3}\right)-\varepsilon<w(t)-\varepsilon<y(t)$. Setting $u(t)=w(t)-\varepsilon, t \geq T_{4}$, we obtain $0<u(t)<y(t), u^{\prime}(t)=w^{\prime}(t)>0, u^{(n-1)}(t)=w^{(n-1)}(t)>0$ and $u^{(n)}(t)=w^{(n)}(t)=-Q(t) G(y(t-\sigma))$. Integrating from $T_{5}$ to $t\left(T_{4}+\sigma<\right.$ $\left.T_{5}<t\right)$ and using $\left(\mathrm{H}_{2}\right)$ we obtain

$$
\begin{aligned}
u^{(n-1)}(t) & =u^{(n-1)}\left(T_{5}\right)-\int_{T_{5}}^{t} Q(s) G(y(s-\sigma)) d s \\
& <u^{(n-1)}\left(T_{5}\right)-G\left(u\left(T_{5}-\sigma\right)\right) \int_{T_{5}}^{t} Q(s) d s .
\end{aligned}
$$

Hence $u^{(n-1)}(t)<0$ for large $t$ by $\left(\mathrm{H}_{1}\right)$, contradicting $u^{(n-1)}(t)>0$ for $t \geq T_{4}$. If $\left(\mathrm{A}_{2}\right)$ is satisfied, then from (5) we get $w\left(t_{n}\right) \geq y\left(t_{n}\right)-F\left(t_{n}\right)$. Hence $\lim _{n \rightarrow \infty} w\left(t_{n}\right)=\infty$. Thus $w(t)>0$ and $w^{\prime}(t)>0$ for $t \geq T_{2}>T_{1}$. Consequently, from (3) it follows that $w^{(n-1)}(t)>0$ for $t \geq T_{3}>T_{2}$. Since $w(t)$ is increasing, we have, for $t>T_{3}+\tau$,

$$
\begin{aligned}
\left(1-p_{2}\right) w(t) & \leq w(t)+p(t) w(t-\tau) \\
& =y(t)-F(t)-p(t) p(t-\tau) y(t-2 \tau)-p(t) F(t-\tau) \\
& \leq y(t)-F(t)-p(t) F(t-\tau) .
\end{aligned}
$$

For $0<\varepsilon<\left(1-p_{2}\right) w\left(T_{3}\right)$, there exists $T_{4}>T_{3}$ such that $|F(t)|<\varepsilon / 2$, $t \geq T_{4}$. From (6) it follows that

$$
\left(1-p_{2}\right) w\left(T_{3}\right)<\left(1-p_{2}\right) w(t) \leq y(t)+\varepsilon / 2-p(t) \varepsilon / 2<y(t)+\varepsilon
$$

for $t>T_{4}+\tau$ because $w(t)$ is increasing and $-p(t)<1$. Setting $u(t)=$ $\left(1-p_{2}\right) w(t)-\varepsilon$ for $t>T_{4}+\tau$, we obtain $0<\left(1-p_{2}\right) w\left(T_{3}\right)-\varepsilon<u(t)<y(t)$, $u^{\prime}(t)=\left(1-p_{2}\right) w^{\prime}(t)>0, u^{(n-1)}(t)=\left(1-p_{2}\right) w^{(n-1)}(t)>0$ and

$$
u^{(n)}(t)=\left(1-p_{2}\right) w^{(n)}(t)=-\left(1-p_{2}\right) Q(t) G(y(t-\sigma)) .
$$


Integrating (7) from $T_{5}$ to $t\left(T_{4}+2 \varrho<T_{5}<t\right)$ we obtain

$$
\begin{aligned}
u^{(n-1)}(t) & <u^{(n-1)}\left(T_{5}\right)-\left(1-p_{2}\right) \int_{T_{5}}^{t} Q(s) G(u(s-\sigma)) d s \\
& <u^{(n-1)}\left(T_{5}\right)-\left(1-p_{2}\right) G\left(u\left(T_{5}-\sigma\right)\right) \int_{T_{5}}^{t} Q(s) d s,
\end{aligned}
$$

because $u$ is increasing. Hence $u^{(n-1)}(t)<0$ for large $t$, due to $\left(\mathrm{H}_{1}\right)$, a contradiction.

Thus $y(t)$ is bounded. Consequently, whether $p(t)$ satisfies $\left(\mathrm{A}_{1}\right)$ or $\left(\mathrm{A}_{2}\right)$, $w(t)$ is bounded and hence

$$
(-1)^{n+k} w^{(k)}(t)<0, \quad k=1, \ldots, n-1,
$$

for $t \geq T_{6}>T_{1}$. If $\liminf _{t \rightarrow \infty} y(t)=\alpha>0$, then $y(t)>\beta>0$ for $t \geq T_{7}$ $>T_{6}$. Hence from (3) we get

$$
w^{(n-1)}(t)<w^{(n-1)}\left(T_{7}+\sigma\right)-G(\beta) \int_{T_{7}+\sigma}^{t} Q(s) d s .
$$

Thus, for large $t, w^{(n-1)}(t)<0$ by $\left(\mathrm{H}_{1}\right)$, contrary to (8). We conclude that $\liminf _{t \rightarrow \infty} y(t)=0$.

Since $w(t)$ is bounded, $\lim _{t \rightarrow \infty} w(t)$ exists due to (8) whether $n$ is odd or even. Hence $\lim _{t \rightarrow \infty} z(t)$ exists. Lemma 2.1 applied to (2) yields $\lim _{t \rightarrow \infty} z(t)$ $=0$.

If $\left(\mathrm{A}_{1}\right)$ holds then for $t \geq T_{1}, z(t) \geq y(t)-p_{1} y(t-\tau)$ implies $y(t) \leq z(t)+$ $p_{1} y(t-\tau)$. Hence $\limsup _{t \rightarrow \infty} y(t) \leq \lim _{t \rightarrow \infty} z(t)+p_{1} \lim _{\sup _{t \rightarrow \infty}} y(t-\tau)$, that is, $\left(1-p_{1}\right) \lim \sup _{t \rightarrow \infty} y(t) \leq 0$. Consequently, $\lim _{t \rightarrow \infty} y(t)=0$.

If $\left(\mathrm{A}_{2}\right)$ holds then $y(t) \leq z(t)$ for $t \geq T_{1}$. Hence $\lim _{t \rightarrow \infty} y(t)=0$. If $y(t)<0$ for $t \geq t_{0}$, then we set $x(t)=-y(t)$ for $t \geq t_{0}$ to obtain

$$
[x(t)-p(t) x(t-\tau)]^{(n)}+Q(t) \widetilde{G}(x(t-\sigma))=\widetilde{f}(t),
$$

where $\widetilde{f}(t)=-f(t)$ and $\widetilde{G}(u)=-G(-u)$. If $\widetilde{F}(t)=-F(t)$, then $\left(\mathrm{H}_{2}\right)$ and $\left(\mathrm{H}_{3}\right)$ are satisfied for $\widetilde{G}$ and $\widetilde{F}$ respectively. Hence $\lim _{t \rightarrow \infty} x(t)=0$, that is, $\lim _{t \rightarrow \infty} y(t)=0$. Thus the theorem is proved.

As a consequence of Theorem 2.2 we get the following.

COROLlary 2.3. Under the assumptions of Theorem 2.2, every nonoscillatory solution of (1) tends to zero as $t \rightarrow \infty$ and hence every unbounded solution of (1) oscillates.

REMARK 2. Theorem 2.2 remains true if $f(t) \equiv 0$.

For sublinear $G$, that is, $G$ satisfying $\int_{0}^{ \pm c} d u / G(u)<\infty, c>0$, or $\liminf _{|u| \rightarrow 0} G(u) / u>\mu>0$, condition $\left(\mathrm{H}_{1}\right)$ cannot be relaxed e.g. to 
$\int_{0}^{\infty} t^{n-1} Q(t) d t=\infty$ used in [5]. This follows from the example given below.

Example. Consider

$$
[y(t)-p y(t-1)]^{\prime \prime}+\frac{3}{8}(t-1)^{-1 / 2}\left(t^{-3 / 2}-p(t-1)^{-3 / 2}\right) y^{1 / 3}(t-1)=0
$$

with either $0<p<1 / 2$ and $t \geq t_{0}>\max \left\{2,\left(1-(2 p)^{2 / 3}\right)^{-1}\right\}$, or $-1<p$ $<0$ and $t>t_{0}>2$. In the first case $\left(\mathrm{H}_{1}\right)$ fails because from the inequality

$$
Q(t)=\frac{3}{8}\left[t^{-3 / 2}(t-1)^{-1 / 2}-p(t-1)^{-2}\right]<\frac{3}{8}\left[t^{-3 / 2}-p(t-1)^{-2}\right]
$$

it follows that $\int_{t_{0}}^{\infty} Q(t) d t<\infty$. However,

$$
Q(t)>\frac{3 p}{8}\left[2(t-1)^{-2}-(t-1)^{-2}\right]=\frac{3 p}{8}(t-1)^{-2}
$$

implies that

$$
\int_{t_{0}}^{t} s Q(s) d s>\frac{3 p}{8} \int_{t_{0}}^{t} s(s-1)^{-2} d s>\ln \left[(t-1) /\left(t_{0}-1\right)\right] .
$$

Hence $\int_{t_{0}}^{\infty} Q(t) d t=\infty$. Similarly in the second case $\int_{t_{0}}^{\infty} Q(t) d t<\infty$ and $\int_{t_{0}}^{t} s Q(s) d s>\frac{3}{8} \int_{t_{0}}^{t} d s / s$ implies that $\int_{t_{0}}^{\infty} t Q(t) d t=\infty$. In both cases, (9) admits a solution $y(t)=t^{3 / 2}$ which is nonoscillatory and unbounded.

In the following theorem, $p(t)$ is allowed to change sign.

Theorem 2.4. Let $\left(\mathrm{H}_{1}\right),\left(\mathrm{H}_{2}\right)$ and $\left(\mathrm{H}_{3}\right)$ hold. Let $p(t)$ be $\tau$-periodic and $\left(\mathrm{A}_{4}\right)$ be satisfied. Then every solution of (1) oscillates or tends to zero as $t \rightarrow \infty$.

Proof. Let $y(t)$ be a nonoscillatory solution of (1). Proceeding as in the proof of Theorem 2.2 we conclude that there is a sequence $\left\{t_{n}\right\}$ satisfying (4). Then $(5)$ and $\left(\mathrm{A}_{4}\right)$ yield

$$
w\left(t_{n}\right) \geq y\left(t_{n}\right)-p_{5} y\left(t_{n}-\tau\right) F\left(t_{n}\right) \geq\left(1-p_{5}\right) y\left(t_{n}\right)-F\left(t_{n}\right) .
$$

Hence $\lim _{n \rightarrow \infty} w\left(t_{n}\right)=\infty$. Thus $w(t)>0$ and $w^{\prime}(t)>0$ for $t \geq T_{2}>T_{1}$. From (3) it follows that $w^{(n-1)}(t)>0$ for $t \geq T_{3}>T_{2}$. Since $w(t)$ is increasing and positive and $p(t)=p(t-\tau)$, we obtain

$$
\begin{aligned}
\left(1-p_{4}\right) w(t) & <w(t)-p_{4} w(t-\tau)<w(t)+p(t) w(t-\tau) \\
& =y(t)-F(t)-p(t) p(t-\tau) y(t-2 \tau)-p(t) F(t-\tau) \\
& =y(t)-F(t)-p^{2}(t) y(t-2 \tau)-p(t) F(t-\tau) \\
& <y(t)-F(t)-p(t) F(t-\tau)
\end{aligned}
$$

for $t \geq T_{4}>T_{3}+\varrho$. Then proceeding as in the proof of Theorem 2.2 we arrive at a contradiction. Hence $y(t)$ is bounded. Consequently, $\lim _{t \rightarrow \infty} z(t)$ exists. Applying the argument of the proof of Theorem 2.2, we may show 
that $\liminf \operatorname{in}_{t \rightarrow \infty} y(t)=0$. As Lemma 2.1 cannot be applied here to show that $\lim \sup _{t \rightarrow \infty} y(t)=0$, we proceed as follows: observe that

$$
\begin{aligned}
\lim _{t \rightarrow \infty} z(t) & =\limsup _{t \rightarrow \infty}[y(t)-p(t) y(t-\tau)] \geq \limsup _{t \rightarrow \infty}\left[y(t)-p_{5} y(t-\tau)\right] \\
& \geq \limsup _{t \rightarrow \infty} y(t)+\liminf _{t \rightarrow \infty}\left[-p_{5} y(t-\tau)\right] \\
& =\limsup _{t \rightarrow \infty} y(t)-p_{5} \limsup _{t \rightarrow \infty} y(t-\tau) \\
& =\left(1-p_{5}\right) \limsup _{t \rightarrow \infty} y(t)
\end{aligned}
$$

and

$$
\begin{aligned}
\lim _{t \rightarrow \infty} z(t) & =\liminf _{t \rightarrow \infty}[y(t)-p(t) y(t-\tau)] \leq \liminf _{t \rightarrow \infty}\left[y(t)+p_{4} y(t-\tau)\right] \\
& \leq \liminf _{t \rightarrow \infty} y(t)+\limsup _{t \rightarrow \infty}\left[p_{4} y(t-\tau)\right]=p_{4} \limsup _{t \rightarrow \infty} y(t-\tau) \\
& =p_{4} \limsup _{t \rightarrow \infty} y(t),
\end{aligned}
$$

since $\liminf \operatorname{in}_{t \rightarrow \infty} y(t)=0$. Combining the above inequalities we get

$$
0 \leq\left(1-p_{5}-p_{4}\right) \limsup _{t \rightarrow \infty} y(t) \leq 0,
$$

because $p_{4}+p_{5}<1$. Hence $\lim _{t \rightarrow \infty} y(t)=0$. If $y(t)<0$ for $t \geq T_{0}$, we obtain $\lim _{t \rightarrow \infty} y(t)=0$ in a similar manner. Thus the theorem is proved.

TheOREM 2.5. Suppose that $\left(\mathrm{H}_{1}\right)-\left(\widetilde{\mathrm{H}}_{4}\right)$ hold and $Q$ is decreasing. If $p(t)$ $\equiv-1$, then every solution of (1) oscillates or tends to zero as $t \rightarrow \infty$.

Proof. Proceeding as in the previous proofs we show that $w(t)$ is monotonic and hence $\lim _{t \rightarrow \infty} w(t)=\ell$, where $-\infty \leq \ell \leq \infty$. If $-\infty \leq \ell<0$ then $z(t)<0$ for large $t$, a contradiction. If $\ell=0$, then $z(t)>y(t)$ implies that $\lim _{t \rightarrow \infty} y(t)=0$. Suppose $0<\ell \leq \infty$. Then $w^{(n-1)}(t)>0$ for large $t$ and hence $\lim _{t \rightarrow \infty} w^{(n-1)}(t)$ exists and is finite. Further, $z(t)>\lambda>0$ for $t \geq T_{2}>T_{1}$. Integrating (3) from $T_{2}$ to $s\left(s>T_{2}\right)$ and then taking the limit as $s \rightarrow \infty$, we obtain

$$
\int_{T_{2}}^{\infty} Q(t) G(y(t-\sigma)) d t<\infty .
$$

On the other hand, for $T_{3}>T_{2}+\varrho, \int_{T_{3}}^{\infty} Q(t) G(z(t-\sigma)) d t \geq G(\lambda) \int_{T_{3}}^{\infty} Q(t) d t$ implies $\int_{T_{3}}^{\infty} Q(t) G(z(t-\sigma)) d t=\infty$ due to $\left(\mathrm{H}_{1}\right)$. Hence

$$
\int_{T_{3}}^{\infty} Q(t)[G(y(t-\sigma))+G(y(t-\sigma-\tau))] d t=\infty
$$

by $\left(\mathrm{H}_{4}\right)$. Consequently, using $(10)$, we obtain $\int_{T_{3}}^{\infty} Q(t) G(y(t-\sigma-\tau)) d t=\infty$, 
that is,

$$
\infty=\int_{T_{3}-\tau}^{\infty} Q(s+\tau) G(y(s-\sigma)) d s<\int_{T_{3}-\tau}^{\infty} Q(s) G(y(s-\sigma)) d s<\infty,
$$

a contradiction. Hence $\ell=0$ is the only possibility. The proof of the theorem is thus complete.

THEOREM 2.6. Let $\left(\mathrm{H}_{2}\right)-\left(\mathrm{H}_{6}\right)$ hold. If $p(t)$ satisfies $\left(\mathrm{A}_{3}\right)$, then every solution of (1) oscillates or tends to zero as $t \rightarrow \infty$. (Observe that $\left(\mathrm{H}_{6}\right) \Rightarrow\left(\mathrm{H}_{1}\right)$ and it is not necessary to assume $Q(t)$ to be decreasing in Theorem 2.6. However, if $Q(t)$ is decreasing then $\left(\mathrm{H}_{6}\right)$ and $\left(\mathrm{H}_{1}\right)$ are equivalent.)

Proof. Using the notations of previous proofs, setting $\lim _{t \rightarrow \infty} w(t)=\ell$, we show that $-\infty \leq \ell<0$ is not possible and $\ell=0$ implies $\lim _{t \rightarrow \infty} y(t)=0$. Assume, if possible, that $0<\ell \leq \infty$. Hence $z(t)>\lambda>0$ and $w^{(n-1)}(t)>0$ for $t \geq T_{2}>T_{1}>T_{0}+\varrho$. If $t \geq T_{3}>T_{2}+\varrho$, then using $\left(\mathrm{H}_{2}\right),\left(\mathrm{H}_{4}\right),\left(\mathrm{H}_{5}\right)$ and $\left(\mathrm{H}_{6}\right)$ we deduce from $(3)$ that

$$
\begin{aligned}
0= & w^{(n)}(t)+Q(t) G(y(t-\sigma)) \\
& \quad+G(-p(t-\sigma))\left[w^{(n)}(t-\tau)+Q(t-\tau) G(y(t-\tau-\sigma))\right] \\
\geq & w^{(n)}(t)+G\left(p_{3}\right) w^{(n)}(t-\tau)+\alpha Q^{*}(t) G(z(t-\sigma)) \\
\geq & w^{(n)}(t)+G\left(p_{3}\right) w^{(n)}(t-\tau)+\alpha G(\lambda) Q^{*}(t) .
\end{aligned}
$$

Integrating the above inequality and using $\left(\mathrm{H}_{6}\right)$ we get

$$
w^{(n-1)}(t)+G\left(p_{3}\right) w^{(n-1)}(t-\tau)<0
$$

for large $t$, a contradiction. The case $y(t)<0$ for $t \geq T_{0}$ may be dealt with similarly. Thus the theorem is proved.

The following example shows that condition $\left(\mathrm{H}_{6}\right)$ cannot be weakened for sublinear $G$.

EXAmple. Consider $(9)$ with $p(t)=-3 / 2$ for $t \geq 2$. We have

$$
Q(t)=(t-1)^{-1 / 2}\left[\frac{3}{8} t^{-3 / 2}+\frac{9}{16}(t-1)^{-3 / 2}\right]
$$

and $Q(t)$ is decreasing. Further, $Q(t)<\frac{15}{16}(t-1)^{-2}$ for $t \geq 2$ implies that $\int_{2}^{\infty} Q(t) d t<\infty$. However, $t Q(t)>\frac{3}{8} t^{-1 / 2}(t-1)^{-1 / 2}>3 /(8 t)$, so $\int_{2}^{\infty} t Q(t) d t=\infty$. Equation (9) admits a positive unbounded solution $y(t)=$ $t^{3 / 2}$.

REMARK 3. In the literature there are a few papers dealing with the case $p(t) \geq 1$ (see $[1,2,5,6])$. In the following some results in this direction are obtained. 
Theorem 2.7. Suppose that $n$ is odd and $\left(\mathrm{H}_{1}\right)$ and $\left(\mathrm{H}_{2}\right)$ hold. If $p(t)$ satisfies $\left(\mathrm{A}_{3}\right)$ then every nonoscillatory solution of

$$
[y(t)-p(t) y(t-\tau)]^{(n)}+Q(t) G(y(t-\sigma))=0
$$

tends to $+\infty$ or $-\infty$ as $t \rightarrow \infty$.

Proof. Proceeding as in the proof of Theorem 2.2, we deduce that

$$
\lim _{t \rightarrow \infty} z^{(n-1)}(t)=\lambda, \quad-\infty \leq \lambda<\infty .
$$

Suppose that $-\infty<\lambda<\infty$.

If $\liminf _{t \rightarrow \infty} y(t)>0$, then $y(t)>\beta>0$ for $t \geq T_{3}>T_{2}$. Hence

$$
\int_{T_{3}+\sigma}^{\infty} Q(t) G(y(t-\sigma)) d t>G(\beta) \int_{T_{3}+\sigma}^{\infty} Q(t) d t
$$

implies that $\int_{T_{3}+\sigma}^{\infty} Q(t) G(y(t-\sigma)) d t=\infty$ by $\left(\mathrm{H}_{1}\right)$. On the other hand, integrating $z^{(n)}(t)+Q(t) G(y(t-\sigma))=0$, we obtain $\int_{T_{3}+\sigma}^{\infty} Q(t) G(y(t-\sigma)) d t$ $=z^{(n-1)}\left(T_{3}+\sigma\right)-\lambda<\infty$, a contradiction. Hence $\liminf _{t \rightarrow \infty} y(t)=0$.

Thus there exists a sequence $\left\{t_{n}\right\} \subset\left[T_{2}, \infty\right)$ such that $t_{n} \rightarrow \infty$ and $y\left(t_{n}\right) \rightarrow 0$ as $n \rightarrow \infty$. As $z\left(t_{n}\right)=y\left(t_{n}\right)-p\left(t_{n}\right) y\left(t_{n}-\tau\right)<y\left(t_{n}\right)$, we have $\lim \sup _{n \rightarrow \infty} z\left(t_{n}\right) \leq 0$. Similarly, $z\left(t_{n}+\tau\right)=y\left(t_{n}+\tau\right)-p\left(t_{n}+\tau\right) y\left(t_{n}\right)>$ $-p_{6} y\left(t_{n}\right)$ implies that $\liminf _{n \rightarrow \infty} z\left(t_{n}+\tau\right) \geq 0$.

If $z(t)>0$ for $t \geq T_{2}$, then $\lim _{t \rightarrow \infty} z(t)=\mu$, where $0 \leq \mu \leq \infty$. If $0<$ $\mu \leq \infty$ then $z(t)>a>0$ for $t \geq T_{4}>T_{2}$. Hence $0 \geq \lim \sup _{n \rightarrow \infty} z\left(t_{n}\right) \geq$ $a>0$, a contradiction. Thus $\mu=0$.

If $z(t)<0$ for $t \geq T_{2}$, then $\lim _{t \rightarrow \infty} z(t)=\mu$, where $-\infty \leq \mu \leq 0$. If $-\infty \leq \mu<0$, then $z(t)<b<0$ for large $t$. Hence $0 \leq \liminf _{n \rightarrow \infty} z\left(t_{n}+\tau\right) \leq$ $b<0$, a contradiction. Thus $\mu=0$.

Consequently, $(-1)^{n+k} z^{(k)}(t)<0, k=0,1, \ldots, n-1$, for large $t$, and $\lim _{t \rightarrow \infty} z^{(k)}(t)=0, k=0,1, \ldots, n-1$. Since $n$ is odd, $z^{\prime}(t)<0$ for large $t$. Hence $z(t)>0$ for $t \geq T_{2}$. From (2) we obtain $y(t)>y(t-\tau)$. Hence $\liminf _{t \rightarrow \infty} y(t)>0$, a contradiction. Thus $\lambda=-\infty$. This implies that $\lim _{t \rightarrow \infty} y(t)=\infty$. Thus the proof of the theorem is complete.

Remark 4 (see [5]). The conclusion of Theorem 2.7 holds for $G$ with $\liminf _{|u| \rightarrow \infty} G(u) / u>\lambda>0$ provided $\left(\mathrm{H}_{1}\right)$ is replaced by $\int_{0}^{\infty} t^{n-1} Q(t) d t$ $=\infty$.

Corollary 2.8. Let the conditions of Theorem 2.7 be satisfied. Then every bounded solution of (11) oscillates.

Theorem 2.9. Suppose that $p(t)$ satisfies $\left(\mathrm{A}_{6}\right)$. Let $\left(\mathrm{H}_{2}\right)$ and $\left(\mathrm{H}_{3}\right)$ hold. If , for every sequence $\left\{\sigma_{i}\right\} \subset(0, \infty)$ such that $\lim _{i \rightarrow \infty} \sigma_{i}=\infty$ and for every 
$\gamma>0$ such that the intervals $\left(\sigma_{i}-\gamma, \sigma_{i}+\gamma\right), i=1,2, \ldots$, are nonoverlapping,

$$
\sum_{i=1}^{\infty} \int_{\sigma_{i}-\gamma}^{\sigma_{i}+\gamma} Q(t) d t=\infty
$$

then every unbounded solution of (1) oscillates or tends to $\pm \infty$ as $t \rightarrow \infty$, and every bounded solution of (1) oscillates or tends to zero as $t \rightarrow \infty$.

Proof. Proceeding as in the proof of Theorem 2.7 we obtain

$$
\lim _{t \rightarrow \infty} w^{(n-1)}(t)=-\infty \quad \text { or } \quad \lim _{t \rightarrow \infty} w^{(n-1)}(t)=\lambda .
$$

If the former holds, then $\lim _{t \rightarrow \infty} w(t)=-\infty$. Since $w(t)>-p_{7} y(t-\tau)$ $-F(t)$, we have $\lim _{t \rightarrow \infty} y(t)=\infty$.

Suppose that the latter holds. Proceeding as in the proof of Theorem 2.7 and using $\left(\mathrm{H}_{3}\right)$, we have $\lim _{t \rightarrow \infty} w(t)=0$. Hence $(-1)^{n+k} w^{(k)}(t)<0, k=$ $0,1, \ldots, n-1$, for $t \geq T_{2}>T_{1}$ and $\lim _{t \rightarrow \infty} w^{(k)}(t)=0, k=0,1, \ldots, n-1$.

If $y(t)$ is unbounded, then there exists a sequence $\left\{t_{n}\right\} \subset\left[T_{2}, \infty\right)$ such that $t_{n} \rightarrow \infty$ and $y\left(t_{n}\right) \rightarrow \infty$ as $n \rightarrow \infty$. Let $\mu>0$. Then $y\left(t_{n}\right)>\mu$ for $n \geq N_{1}>0$. From the continuity of $y$ it follows that there exists $\delta_{n}>0$ with $\liminf _{n \rightarrow \infty} \delta_{n}>0$ such that $y(t)>\mu$ for $t \in\left(t_{n}-\delta_{n}, t_{n}+\delta_{n}\right)$. Then choosing $n$ large enough such that $\delta_{n}>\delta>0$ for $n \geq N>N_{1}$, we obtain

$$
\begin{aligned}
\int_{T_{2}}^{\infty} Q(t) G(y(t-\sigma)) d t & \geq \sum_{n=N}^{\infty} \int_{t_{n}-\delta_{n}+\sigma}^{t_{n}+\delta_{n}+\sigma} Q(t) G(y(t-\sigma)) d t \\
& >G(\mu) \sum_{n=N}^{\infty} \int_{t_{n}-\delta_{n}+\sigma}^{t_{n}+\delta_{n}+\sigma} Q(t) d t \\
& >G(\mu) \sum_{n=N}^{\infty} \int_{t_{n}-\delta+\sigma}^{t_{n}+\delta+\sigma} Q(t) d t .
\end{aligned}
$$

From the hypothesis it follows that $\int_{T_{2}}^{\infty} Q(t) G(y(t-\sigma)) d t=\infty$. Integrating (3) yields

$$
\int_{T_{2}}^{\infty} Q(t) G(y(t-\sigma)) d t=w^{(n-1)}\left(T_{2}\right)<\infty
$$

a contradiction.

If $y(t)$ is bounded, then we claim $\lim \sup _{t \rightarrow \infty} y(t)=0$. If not, then $\lim \sup _{t \rightarrow \infty} y(t)=\alpha, \alpha>0$. Then there exists a sequence $\left\{t_{n}\right\}$ such that $y\left(t_{n}\right)>\beta>0$ for large $n$. Proceeding as above we arrive at a contradiction. Hence our claim holds. Consequently, $\lim _{t \rightarrow \infty} y(t)=0$. The case $y(t)<0$ for $t \geq T_{0}$ may be dealt with similarly. Thus the theorem is proved. 
Acknowledgements. The authors are grateful to the referee for various suggestions to improve the quality of the paper.

\section{References}

[1] M. P. Chen, Z. C. Wang, J. S. Yu and B. G. Zhang, Oscillation and asymptotic behaviour of higher order neutral differential equations, Bull. Inst. Math. Acad. Sinica 22 (1994), 203-217.

[2] Q. Chuanxi and G. Ladas, Oscillations of higher order neutral differential equations with variable coefficients, Math. Nachr. 150 (1991), 15-24.

[3] I. Győri and G. Ladas, Oscillation Theory of Delay-Differential Equations with Applications, Clarendon Press, Oxford, 1991.

[4] T. H. Hildebrandt, Introduction to the Theory of Integration, Academic Press, New York, 1963.

[5] N. Parhi and R. N. Rath, On oscillation of solutions of forced nonlinear neutral differential equations of higher order, Czechoslovak Math. J., in press.

[6] D. Q. Tang, Oscillation of higher order nonlinear neutral functional-differential equation, Ann. Differential Equations 12 (1996), 83-88.

Department of Mathematics

Berhampur University

Berhampur 760007, Orissa, India

E-mail: parhi2002@rediffmail.com
Department of Mathematics Khallikote (Autonomous) College Berhampur 760001, Orissa, India E-mail: radhanathmath@yahoo.co.in 